\title{
Espectroscopia Anelástica em Ligas de Nb-16\% p. Ti
}

\author{
Renata Abdallah Nogueira ${ }^{\mathrm{a}}$, Terlize Cristina Niemeyer ${ }^{\mathrm{a}, \mathrm{b}}$ \\ Carlos Roberto Grandini ${ }^{\mathrm{a}}$, Odila Florêncio ${ }^{\mathrm{c}}$ \\ ${ }^{a}$ Departamento de Física, Universidade Estadual Paulista \\ 17033-360 Bauru - SP, Brazil \\ ${ }^{\mathrm{b}}$ Instituto de Física de São Carlos, Universidade de São Paulo \\ 13560-970 São Carlos - SP, Brazil \\ ${ }^{\mathrm{c}}$ Departamento de Física, Universidade Federal de São Carlos \\ 13565-905 São Carlos - SP, Brazil
}

Received: April 25, 2003; Revised: August 31, 2003

\begin{abstract}
Átomos de oxigênio, presentes em metais com estrutura cristalina cúbica de corpo centrado, são localizados preferencialmente em sítios octaedrais. Na ausência de uma tensão mecânica externa, estes átomos são distribuídos aleatoriamente. Na presença de oscilações mecânicas, a redistribuição dos átomos nos diferentes sítios deve ocorrer pela interação com esta tensão, levando a perdas na energia elástica. Este trabalho mostra o estudo da interação de átomos de oxigênio presentes em amostras da liga $\mathrm{Nb}-16 \%$ p. Ti, utilizando um pêndulo de torção. Os resultados mostram espectros bastante complexos, que foram decompostos em seus picos constituintes, representando os processos de relaxação devido à reorientação induzida por tensão de átomos e pares de átomos de oxigênio em torno de átomos de nióbio da matriz metálica e átomos de oxigênio em torno de átomos de titânio (interação substitucional-intersticial).
\end{abstract}

Palavras-Chave: atrito interno, difusão, intersticiais, ligas de $\mathrm{Nb}-\mathrm{Ti}$

\section{Introdução}

Átomos de impurezas intersticiais como oxigênio, nitrogênio, carbono e hidrogênio presentes em metais com estrutura cristalina cúbica de corpo centrado, tais como nióbio, vanádio, tântalo e suas ligas, alteram de maneira significativa as propriedades mecânicas destes metais e ligas. Em estruturas cristalinas deste tipo, tais elementos intersticiais localizam-se geralmente em sítios octaedrais. Na presença de uma tensão mecânica externa, estes elementos intersticiais se redistribuem nos diferentes sítios causando perda na energia elástica, difundindo-se na matriz metálica por meio de saltos entre sítios energeticamente equivalentes, causando distorções locais. A interação destes elementos intersticiais com os metais do grupo $\mathrm{V}$ tem sido bastante estudada por meio de medidas de atrito interno em função da temperatura ${ }^{1-4}$.

Em metais que apresentam átomos de impurezas dissolvidos intersticialmente em sua estrutura, observam-se picos no espectro de atrito interno em função da temperatura.
Cada espécie de átomos de soluto intersticial dá origem a um máximo no espectro da perda da energia elástica. Os princípios da natureza elástica deste fenômeno foram estabelecidos por Snoek $^{5}$ que postulou que estes átomos reorientam-se sob a ação de uma tensão externa aplicada. $\mathrm{O}$ pico no espectro de atrito interno em função da temperatura devido a esta reorientação ficou conhecido como Pico de Snoek.

Em ligas com baixa concentração de intersticiais, o pico de Snoek é muito bem ajustado utilizando-se as equações de Debye $^{6}$, no qual a largura a meia altura descreve a reorientação termicamente ativada de átomos isolados do soluto. Quando há agrupamentos de dois ou mais átomos intersticiais estes interagem entre si causando um alargamento da estrutura de relaxação, representado pela superposição de vários picos, um para cada tipo de interação.

Este trabalho mostra o estudo da interação de átomos de oxigênio presentes numa amostra da liga Nb-16\% p. Ti por meio de medidas de espectroscopia anelástica (atrito interno).

*e-mail: betog@ @fc.unesp.br

Trabalho apresentado no XV CBECIMAT, Natal - RN, Novembro de 2002. 


\section{Parte Experimental}

As amostras da liga $\mathrm{Nb}-16 \%$ p. Ti, foram produzidas por fusão por feixe de elétrons e zona flutuante, no Departamento de Engenharia de Materiais da Faculdade de Engenharia Química (FAENQUIL) de Lorena (SP). Estas amostras contêm oxigênio intersticial, provavelmente residual do processo de fusão. Por intermédio de uma análise química, a quantidade de oxigênio foi estimada em $(0,15 \pm 0,02) \%$ em peso.

As medidas de atrito interno e freqüência foram realizadas em um Pêndulo de Torção invertido do tipo $\mathrm{Kê}^{7}$, na faixa de temperatura entre 290 e $700 \mathrm{~K}$ e uma freqüência de oscilação variando entre 0,7 e 4,0 Hz. As medidas foram efetuadas com uma taxa de aquecimento de aproximadamente $1 \mathrm{~K} / \mathrm{min}$, sob vácuo próximo de $10^{-6} \mathrm{mbar}$.

Para a obtenção dos dados de atrito interno foi utilizado o seguinte princípio: a amostra foi colocada a vibrar em seu estado fundamental (vibrações livres ou forçadas), causando dissipação de energia sob a forma de calor, devido ao atrito interno. No pêndulo de torção, esta dissipação de energia por ciclo é medida pelo decremento logarítmico da amplitude das oscilações livres do sistema, que é, a menos de uma constante, o próprio atrito interno da amostra. Os dados relativos ao amortecimento da amplitude de oscilação foram coletados automaticamente, utilizando-se um feixe de laser refletido por um espelho colocado sobre o eixo do pêndulo e atingindo dois fotodiodos conectados, por intermédio de uma interface, a um microcomputador. Deste modo é possível a obtenção de dados a cada 0,5 grau de variação da temperatura. Em nosso caso, os dados foram obtidos a cada $1,0^{\circ}$ de variação da temperatura.

\section{Resultados e Discussão}

Um espectro típico de atrito interno e freqüência em função da temperatura é mostrado na Fig. 1 para uma amostra da liga $\mathrm{Nb}-16 \%$ p. Ti, medido com frequiência de $1,43 \mathrm{~Hz}$, no intervalo de temperatura compreendido entre 290 e 700 K. Aqui, observamos a presença de uma estrutura de relaxação (pico) complexa, provavelmente composta por processos de relaxação que poderão ser atribuídos à reorientação induzida por tensão de átomos de oxigênio em torno de átomos de nióbio e em torno de átomos de titânio da matriz metálica.

Para verificar a veracidade desta suposição, a amostra foi submetida a medidas de relaxações anelásticas a outras freqüências. A Fig. 2 mostra o espectro de atrito interno em função da temperatura, medido com frequiências de aproximadamente 0,$76 ; 1,43$ e 3,45 Hz. Podemos observar na figura, que há um deslocamento da estrutura de relaxação para a região de temperatura mais alta, mostrando que o processo é termicamente ativado.

$\mathrm{O}$ atrito interno causado pela reorientação induzida por

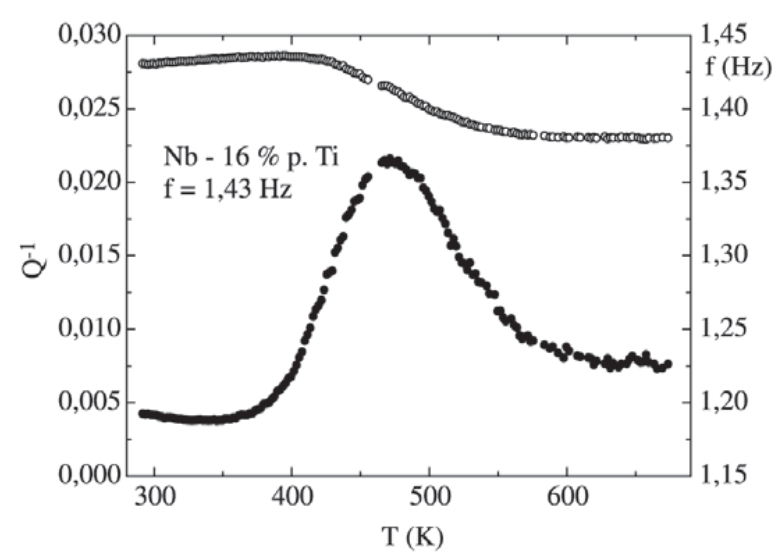

Figura 1. Atrito interno e frequiência em função da temperatura, para a amostra de $\mathrm{Nb}-16 \%$ p. Ti contendo oxigênio, medida com frequiência de 1,43 Hz.

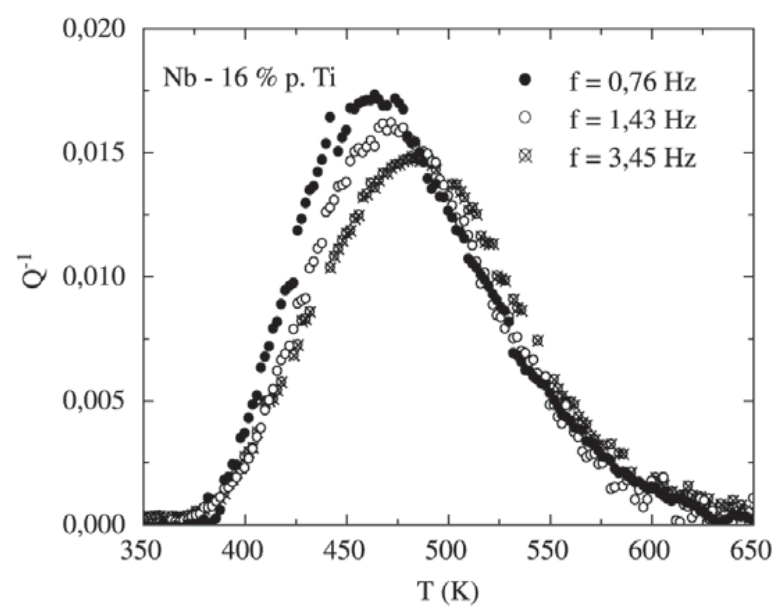

Figura 2. Influência da freqüência na estrutura de relaxação anelástica observada na amostra da liga $\mathrm{Nb}-16 \%$ p. Ti contendo oxigênio em solução sólida.

tensão de átomos intersticiais, em uma matriz metálica, pode ser descrito pela equação de Debye ${ }^{2}$ :

$$
Q^{-1}(\omega \tau)=\Delta \frac{\omega \tau}{1+\omega^{2} \tau^{2}}
$$

onde $\Delta$ é a intensidade de relaxação, $\omega$ é a freqüência angular de oscilação do pêndulo e $\tau$ o tempo de relaxação do processo envolvido.

$\mathrm{O}$ atrito interno e a frequiência medidos nas proximidades do pico de Snoek, em função da temperatura, constituem uma ferramenta que pode ser utilizada para a obtenção da entalpia de ativação e da freqüência de reorientação, do processo de relaxação, pela Lei de Arrhenius ${ }^{6}$. Vamos su- 
por inicialmente que a estrutura de relaxação seja devido a um único processo de relaxação. A condição básica para a ocorrência de um pico de relaxação, associado a um único processo de relaxação é:

$$
\omega \tau=1
$$

onde: $\tau$ é o tempo de relaxação do processo, $\omega=2 \pi \mathrm{f}$ é a freqüência angular e f é a freqüência de oscilação do pêndulo.

Assim:

$$
\ln \mathrm{f}=-\ln 2 \pi \tau_{0}-\mathrm{H} / \mathrm{kT}_{\mathrm{p}}
$$

onde: $T_{p}$ é a temperatura do pico e $\tau_{\mathrm{o}}$ é o tempo de relaxação fundamental do processo.

Então, se fizermos um gráfico do logaritmo da freqüência em função do inverso da temperatura do pico, podemos obter a entalpia de ativação e o tempo de relaxação fundamental do processo. A Fig. 3 mostra tal gráfico. Por meio de uma análise de regressão linear, obtivemos a entalpia de ativação e a freqüência de reorientação, admitindo que o processo de relaxação em questão possa ser representado por um único pico de relaxação:

$$
\mathrm{H}=(1,6 \pm 0,2) \mathrm{eV} \quad \tau_{\mathrm{o}}^{-1}=(2,0 \pm 0,1) \times 10^{17} \mathrm{~s}^{-1}
$$

Os resultados obtidos neste trabalho estão em discordância com os resultados apresentados na literatura ${ }^{8-11}$. Desta forma, podemos concluir inicialmente, que o pico em questão não é somente devido à reorientação induzida por tensão de átomos de oxigênio em torno de átomos de nióbio da matriz metálica. As discrepâncias são devido à existência de picos

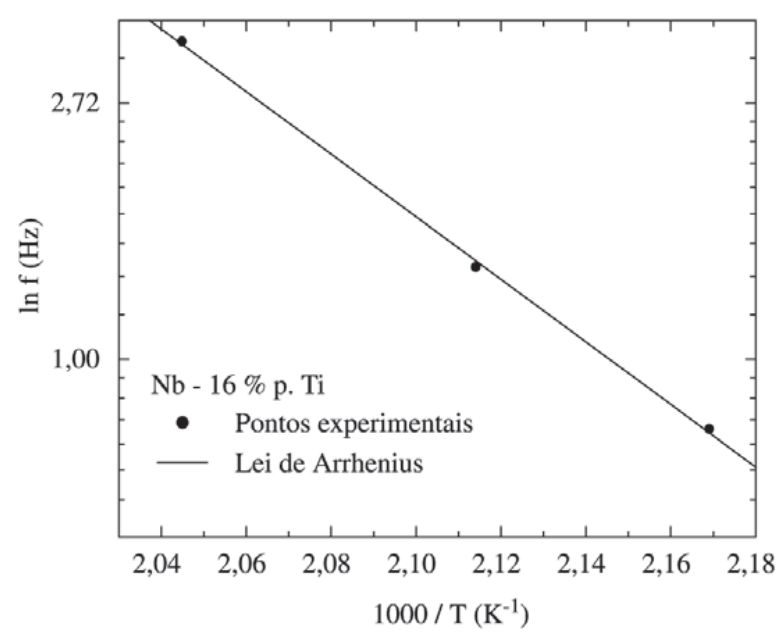

Figura 3. Logaritmo da freqüência em função do inverso da temperatura para o processo de relaxação observado na amostra da liga $\mathrm{Nb}-16 \%$ p. Ti. causados pelo processo de relaxação devido à reorientação induzida por tensão de átomos de oxigênio em torno de átomos de titânio da matriz metálica e à relaxação de pares de intersticiais em torno de átomos de nióbio.

Para confirmar a conclusão acima, vamos usar um modelo proposto por Weller et al. ${ }^{3}$, utilizando a Eq. 1 combinada com a Lei de Arrhenius, adicionando a dependência da temperatura para a intensidade de relaxação $\Delta$ (proporcional a $1 / \mathrm{T}$ ) e a correção na frequiência a freqüência muda com a temperatura por que o módulo elástico depende da temperatura e é proporcional a $\mathrm{f}^{2}(\mathrm{~T})$, o atrito interno pode ser escrito como $^{6}$ :

$$
Q^{-1}(T)=Q_{m}^{-1} \frac{T_{m}}{T} \cosh ^{-1}\left[\frac{H}{k_{B}}\left(\frac{1}{T}-\frac{1}{T_{m}}\right)+\ln \left(\frac{f(T)}{f_{m}}\right)\right]
$$

Os três parâmetros que caracterizam um máximo de Debye são $\mathrm{Q}^{-1}{ }_{\mathrm{m}}$ (altura do pico), $\mathrm{T}_{\mathrm{m}}$ (temperatura do pico) e $\mathrm{H}$ (entalpia de ativação do processo), podendo ser determinadas aplicando métodos apropriados de análise numérica para a interpretação dos dados experimentais (no presente trabalho foi usado o Modulo PeakFitting do Microcal $^{\mathrm{M}}$ Origin ${ }^{\circledR}$ ).

Quando mais de um processo de relaxação está presente, um comportamento mais complicado é esperado e interações de defeitos podem alargar os picos devido aos vários tempos de relaxação. Se vários tipos de defeitos com diferentes entalpias estão presentes, então uma superposição de vários picos de Debye é esperada e o atrito interno pode ser escrito por:

$$
Q^{-1}(T)=\sum_{i=1}^{n} Q_{m i}^{-1} \frac{T_{m i}}{T} \cosh ^{-1}\left[\frac{H_{i}}{k_{B}}\left(\frac{1}{T}-\frac{1}{T_{m i}}\right)+\ln \left(\frac{f(T)}{f_{m i}}\right)\right]
$$

Usando um método de regressão não-linear baseado num processo de subtração sucessiva, analisamos as curvas presentes na Fig. 2. Três processos de relaxação anelástica foram propostos: a reorientação induzida por tensão dos átomos de oxigênio em torno de átomos de nióbio ( $\mathrm{Nb}-\mathrm{O})$; a reorientação induzida por tensão de pares de átomos de oxigênio em torno de átomos de nióbio (Nb-O-O) e a reorientação induzida por tensão de átomos de oxigênio em torno de átomos de titânio (Ti-O), descrevendo o processo de interação substitucional-intersticial. As entalpias de ativação para os três processos propostos foram fixadas, baseando-se em valores determinados por experimentos de atrito interno ${ }^{9-11}$, isto é, $1,15,1,18$ e 1,23 eV, respectivamente. As Figs. 4 e 5 apresentam a decomposição mencionada acima para a amostra medida com a freqüência inicial de 0,76 e 1,43 Hz, respectivamente, na qual a linha cheia representa 


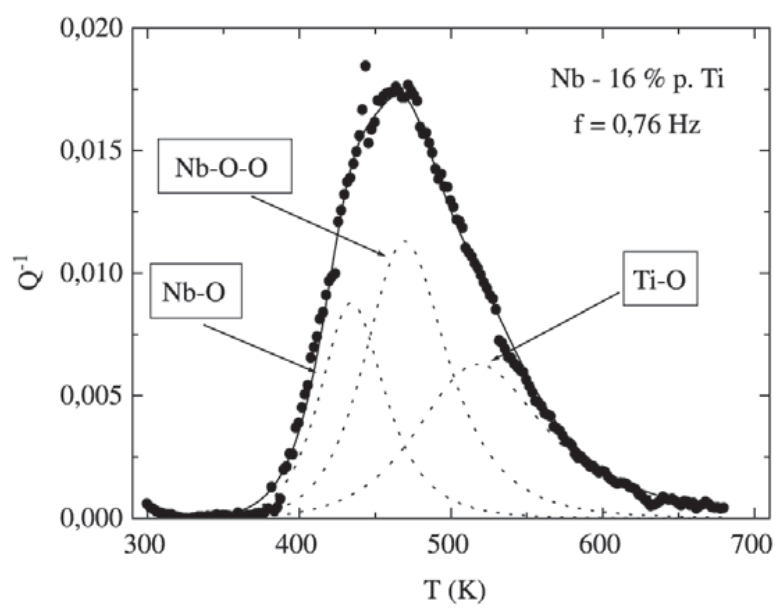

Figura 4. Decomposição em picos de Debye do espectro de atrito interno em função da temperatura para a liga $\mathrm{Nb}-16 \%$ p. Ti, medido com freqüência inicial de $0,76 \mathrm{~Hz}$.

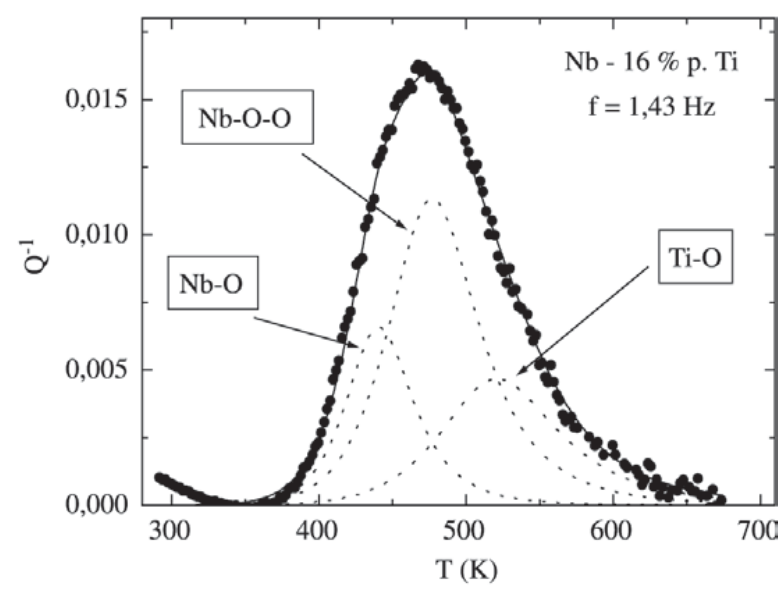

Figura 5. Decomposição em picos de Debye do espectro de atrito interno em função da temperatura para a liga $\mathrm{Nb}-16 \%$ p. Ti, medido com freqüência inicial de 1,43 Hz.

a soma dos três picos de Debye (linha pontilhada), que representam os três processos de relaxação propostos. Os parâmetros de relaxação anelástica $\left(\mathrm{Q}^{-1}{ }_{\mathrm{m}}, \mathrm{H}\right.$ e $\left.\mathrm{T}_{\mathrm{m}}\right)$ para os três processos são representados na Tabela 1 , na qual são apresentados os valores das freqüências iniciais de oscilação do pêndulo de torção (valores obtidos para a temperatura de $300 \mathrm{~K}$ ).

\section{Conclusões}

Foi analisada, por intermédio das medidas de espectroscopia anelástica, uma amostra da liga $\mathrm{Nb}-16 \%$ p. Ti contendo oxigênio em solução sólida.
Tabela 1. Parâmetros para os processos de relaxação devido ao oxigênio na liga $\mathrm{Nb}-16 \%$ p.Ti.

\begin{tabular}{ccccc}
\hline Processo & $\mathrm{f}(\mathrm{Hz})$ & $\mathrm{T}_{\mathrm{m}}(\mathrm{K})$ & $\mathrm{H}(\mathrm{eV})$ & $\mathrm{Q}^{-1}{ }_{\mathrm{m}}$ \\
\hline \multirow{2}{*}{$\mathrm{Nb}-\mathrm{O}$} & 0,76 & 434 & 1,15 & 0,008 \\
& 1,43 & 440 & 1,15 & 0,008 \\
\multirow{2}{*}{ Nb-O-O } & 0,76 & 469 & 1,18 & 0.011 \\
& 1,43 & 477 & 1,18 & 0.011 \\
\multirow{2}{*}{ Ti-O } & 0,76 & 519 & 1,23 & 0.006 \\
& 1,43 & 526 & 1,23 & 0.006 \\
\hline
\end{tabular}

Os resultados mostraram estruturas de relaxação termicamente ativadas que foram decompostas em seus picos constituintes, associados aos processos de relaxação devido à reorientação induzida por tensão de átomos de oxigênio em torno de átomos de nióbio (processo $\mathrm{Nb}-\mathrm{O}$ ), pares de átomos de oxigênio em torno de átomos de nióbio (processo Nb-O-O) e a interação substitucional-intersticial, devido à reorientação induzida por tensão de átomos de oxigênio em torno de átomos de titânio (processo Ti-O).

\section{Agradecimentos}

Os autores agradecem ao Departamento de Materiais (DEMAR) da Faculdade de Engenharia Química de Lorena (FAENQUIL), pelas amostras, à Fundação de Amparo à Pesquisa do Estado de São Paulo (FAPESP), ao Conselho Nacional de Desenvolvimento Científico e Tecnológico (CNPq) e à Fundação para o Desenvolvimento da UNESP (FUNDUNESP) pelo suporte financeiro.

\section{Referências}

1. Cantelli, R.; Szkopiak, Z.C. Appl. Phys., v. 9, p. 153, 1976.

2. Haneczok, G.; Weller, M. J. Less-Common Metals, v. 159, p. 269, 1990.

3. Weller, M.; Haneczok, G.; Diehl, J. Phys. Stat. Sol. B 172, v. 145, 1992.

4. Grandini, C.R.; Botta Filho, W.J.; Florêncio, O.; Tejima, H.; Jordão, J.A.R. J. Alloys and Comp. 211, v. 226, 1994.

5. Snoek, J.L. Physica 8, v. 711, 1941.

6. Nowick, A.S.; Berry, B.S. Anelastic Relaxation in Crystalline Solids, Academic Press, New York, 1972.

7. Kê, T.S. Phys. Rev.71, v. 533, 1947.

8. Florêncio, O.; Grandini, C.R.; Botta Filho, W.J.; Tejima, H.; Jordão, J.A.R. J. Alloys and Comp. 211, v. 37, 1994.

9. Weller, M. J. Physique IV 5, C7-199, 1995.

10. Grandini, C.R.; Ferreira, L.E.C.; Sandim, H.R.Z.; Florêncio, O.; Tejima, H.; Jordão, J.A.R. J. Physique IV 6, C8-135, 1996.

11. Niemeyer, T.C.; Gimenez, J.M.A.; Almeida, L.H.; Grandini, C.R.; Florêncio, O. Mat. Res. 5, v. 143, 2002. 